\title{
Why Betas Should Not Rule Metas
}

\author{
Cort W. Rudolph ${ }^{\star}$ \\ Saint Louis University
}

\author{
Dustin K. Jundt \\ Saint Louis University
}

The inclusion of partial regression coefficients in the Ferguson (2015a) meta-analysis published in Perspectives on Psychological Science caught our eye. While some anecdotal support was given in this paper to justify this practice, no empirical rationale was offered. This is odd, because a great deal of methodological literature cautions against this practice. To better address this concern, we present evidence through both computational examples and Monte Carlo simulations to suggest that the inclusion of partial relationships in meta-analytic models represents a statistical misspecification that obfuscates the ability to estimate the true population effect size. Additionally, we discuss three means of remediating this issue that specifically address the possibility of statistical control in meta-analysis.

Keywords. Meta-Analysis, Statistical Control, Monte Carlo Simulation

The recent back-and-forth regarding the Ferguson (2015a) meta-analysis peaked our attention, particularly the arguments offered by Ferguson (2015b) for the inclusion of partial regression coefficients in meta-analysis. Indeed, consistent with Boxer, Groves, and Dochert (2015) and Rothstein and Bushman (2015), we were somewhat surprised by this assertion. Specifically, we understand it to be axiomatic that one should not include partial coefficients in meta-analysis, and a quick review of various well-regarded resources on the subject highlights the degree of caution against this practice that is present in the literature (e.g., Lipsey \& Wilson, 2001, p. 67; Cooper, 2010, p. 192; Schmidt \& Hunter, 2015, p. 495). Nevertheless, this practice has been justified by Ferguson (2015b) by citing previous works, which have suggested that partial relationships are generally of a lower magnitude than zero order relationships (e.g., Pratt et al., 2010 p. 776). Moreover, this "smaller effects" rationale is used as justification for the inclusion of such parameters in meta-analytic models, because of the assumption that these relationships have been in some way "refined" or made to be more conservative by virtue of statistical control. The arguments follows that partial coefficients are thus a better representation of reality (i.e., the population parameter) because they account for possibly spurious zero order effects.

Unfortunately, this is simply not true. Let us explore why.

\section{The Problem Understood Through a Computational Example.}

To demonstrate why, we will first explore a quick computational example. Formula 3.3.11 from Cohen, Cohen, West, \& Aiken (2003) allows one to decompose a three-by-three correlation matrix into partial relationships:

$$
\text { [1] } r_{\text {partial }}=\frac{r_{Y 1}-r_{Y 2} r_{12}}{\sqrt{1-r_{Y 2}^{2}} \sqrt{1-r_{12}^{2}}}
$$

\footnotetext{
${ }^{\star}$ Cort W. Rudolph \& Dustin K. Jundt, Department of Psychology, Saint Louis University, St. Louis, MO (USA). Correspondence concerning this article should be addressed to Cort Rudolph, Saint Louis University, Morrissey Hall 2827 St. Louis, MO, 63103, rudolphc@slu.edu, +1(314) 977-7299. Portions of this manuscript were presented at the 2016 annual conference of the Association for Psychological Science, Chicago IL (USA).
} 
For the purposes of this example, let us assume two cases (i.e., Case \#1 \& Case \#2; see table 1) that define the intercorrelations between an independent (IV) and dependent (DV) variable and a single covariate (COV).

Table 1. Case \#1 and Case \#2 for Deriving Partial Correlations

\begin{tabular}{|c|c|c|c|c|c|c|c|}
\hline \multicolumn{4}{|c|}{ Case \#1 } & \multicolumn{4}{|c|}{ Case \#2 } \\
\hline & IV & $\mathrm{COV}$ & DV & & IV & $\mathrm{COV}$ & DV \\
\hline IV & 1.00 & & & IV & 1.00 & & \\
\hline $\mathrm{COV}$ & 0.50 & 1.00 & & $\mathrm{COV}$ & 0.50 & 1.00 & \\
\hline DV & 0.25 & 0.50 & 1.00 & DV & 0.25 & 0.00 & 1.00 \\
\hline
\end{tabular}

Notice that Case \#1 and Case \#2 differ only by the strength of the relationship between the independent variable and covariate and the dependent variable and the covariate. That is to say, the parameter defining the IV-DV relationship is the same $\left(r_{x y}=.25\right)$. That said, if we apply formula one, above, then Case \#1 yields a partial correlation $r_{\text {partial }}=0.00$ (i.e., an underestimate of the zero-order effect), whereas Case \#2 yields $r_{\text {partial }}=0.29$ (i.e., an overestimate of the zeroorder effect). Simply put, partial relationships can, in various cases, either over or under estimate a zero-order effect.

Why do we get such different results with the partial effect when compared to the zero order effect? The nature of partial effects -- such as standardized regression coefficients (i.e., "Betas" - which in the three-variable case presented here is mathematically equivalent to the partial correlation coefficient) -- is that they account for shared variance associated with a covariate that is partialled out of both the independent and the dependent side of the regression equation (See Cohen, Cohen, West, \& Aiken, 2003 p. 72). In terms of understanding main effect relationships, the two partial correlations calculated above would lead to very different inferences compared to the known value of the zero order relationship.

\section{The Problem Understood Through Monte Carlo Simulation.}

Whether or not this is really an issue for meta-analytic conclusions bears further empirical elaboration. This is particularly important, and the evidence offered by Ferguson $(2015 \mathrm{a}, \mathrm{b})$ for the inclusion of partial effects in meta-analysis comes only from previous studies that have adopted the same practice (e.g. Paternoster, 1987; Pratt et al., 2010; Savage \& Yancey, 2008). If the inclusion of partial relationship in meta-analysis represents a potential misspecification error as we suspect that it does, then conclusions drawn from such analyses may be questionable, and should be subject to further scrutiny.

To address this, we first conducted a Monte Carlo simulation ${ }^{1}$ to gauge whether or not the inclusion of partial relationships in meta-analysis would in some way affect the conclusions one would draw. We structured this simulation as such. First, to be consistent with our example above, we assumed that the parameters defined by Case \#1 and Case \#2 represent "true"

\footnotetext{
${ }^{1}$ An $\mathrm{R}$ program to replicate the simulations presented here is available from the first author.
} 
population correlation matrices of interest. That is to say, the $\rho_{x y}=.25$ in both cases is a parameter that is fixed in the population, as are the $\rho_{c y}$ and $\rho_{x c}$ effects.

For both Case \#1 and Case \#2, we first simulated one hundred 10,000 person "populations" that conform to this correlation structure, and from this we randomly selected one hundred samples of 250. Each of these samples constitutes a simulated $N=250$ "study" that was drawn from each population. Importantly, the value of the correlation parameters was fixed within each population. Thus, variability observed in these effects between each study can be attributed to sampling error. This is important, because one of the primary goals of meta-analysis is to overcome sampling error to arrive at "truer" estimates of population parameters. For each study, we derived a zero-order correlation reflecting the independent-dependent variable relationship, and a partial correlation reflecting the independent-dependent variable relationship, controlling for the covariate. Then, for each case, we subjected both the zero order and the partial correlations derived from the 100 simulated studies to a meta-analysis. Consistent with our approach of randomly sampling from multiple hypothetical populations, we followed randomeffects estimation procedures (Dersimonian-Laird), and derived an average sample-weighted effect size estimate (i.e., $\rho$ ) along with an associated standard error to construct $95 \%$ confidence intervals for each relationship (i.e., zero-order and partial). Table 2 summarizes the results of this procedure for Case \#1 and Case \#2, and Figures $1 \& 2$ plot the effect sizes derived for each study to visually represent the distribution of estimate parameters.

Table 2. Results of Meta-Analyses for Zero-Order and Partial Effects for Case \#1 \& Case \#2

\begin{tabular}{llccc}
\hline Case \#1 & & $\rho$ & $95 \%$ CI Lower & 95\% CI Upper \\
\hline & Zero Order & 0.253 & 0.236 & 0.259 \\
& Partial & -0.001 & -0.013 & 0.012 \\
\hline Case \#2 & & $\rho$ & $95 \%$ CI Lower & $95 \%$ CI Upper \\
\hline & Zero Order & .251 & .238 & .263 \\
& Partial & .335 & .321 & .345 \\
\hline
\end{tabular}

Note. $\rho$ denotes meta-analytic effect size estimate. $\mathrm{K}=100, \mathrm{~N}=25,000$

From this simulation, it is quite clear that one would reach very different conclusions regarding the independent-dependent variable relationship depending on whether one considered a metaanalysis of zero-order or partial effects. Moreover, just as we saw in our computational example, depending on the nature of the relationship between covariates and other variables in the model, one's conclusions may vastly under or overestimate the population parameter. It is critical to note the meta-analyses of partial effects reported here represent hypothetical cases where the same (presumably theory-driven) control variable is included in each study. Given numerous criticisms of the application of statistical control techniques in psychology and related fields (e.g. Becker, 2005; Breaugh, 2008; Meehl, 1971; Spector \& Brannick, 2011), it is likely an unsafe assumption that the partial relationships reported in studies of a given topic use control variables in such a systematic manner.

In many ways, this simulation serves to highlight a "worst case" scenario in which meta-analyses entirely composed of zero-order versus partial relationships are being compared. Perhaps a more realistic scenarios is that a meta-analyst includes some percentage of partial relationships in the 
overall analysis to either attempt to account for a confounding factor (i.e., as was the case in the Ferguson, 2015a) or because zero-order information was not reported in a portion of primary studies. This begs the question, "To what extent does the inclusion of varying ratios of zeroorder to partial effects bias the conclusions one would draw from a meta-analysis?" We again turn to a Monte Carlo simulation to answer this question.

Figure 1. Scatter Plot - Case \#1

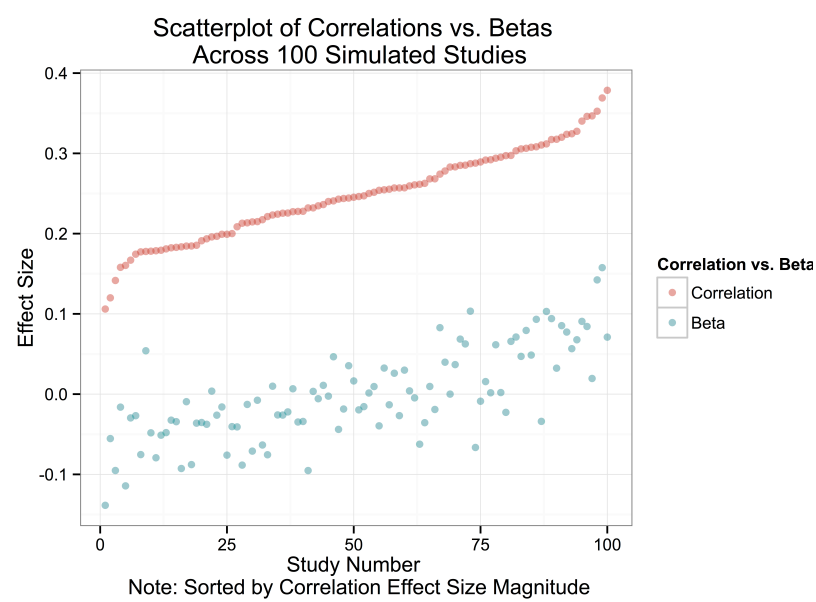

Figure 2. Scatter Plot - Case \#2

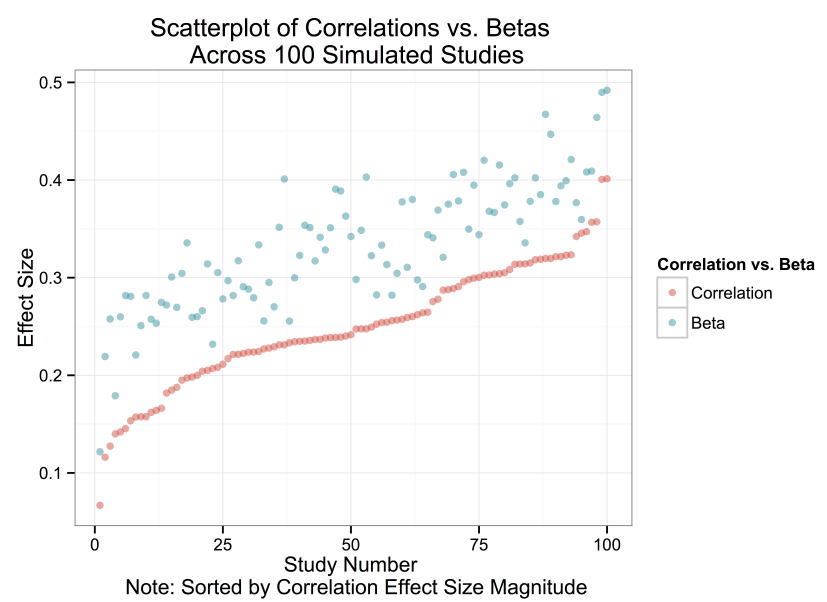

The basic design of the second simulation was similar to the first, except in this case we sampled varying ratios of zero-order to partial correlations prior to conducting each meta-analysis. We did this for various "mixes" of effects in increments of $10 \%$ (i.e., ranging from $90 \%$ zero-order $/ 10 \%$ partial to $10 \%$ zero-order $/ 90 \%$ partial). Table 3 displays the results of each of 9 meta-analyses conducted with these variable mixes for both Case \#1 and Case \#2, while Figures 3 \& 4 graphically displays the results of this simulation for Case \#1 and Case \#2, respectively.

Considering the results of this simulation, it is clear that bias (i.e., with respect to accurately capturing the population parameter in one's meta-analytic estimate of this effect) is systematically introduced when even small numbers (i.e., 10\%, in this case 10 studies out of 100) of partial relationships are included. To us, this signals the fact partial relationships should not "rule" meta-analysis, and in fact should never be considered when constructing meta-analytic estimates.

\section{Three Solutions for the Concern of Statistical Control in Meta-analysis.}

While the preceding highlights one substantial concern with including partial coefficients in meta-analysis, there are some potential remediating procedures for the issue of statistical control in meta-analysis that bear consideration here. First, one could code study-level confounds as moderators (e.g., To ask a question such as, "Are study effects stronger/weaker among experimental vs. quasi-experimental vs. cross-sectional studies?"). This would allow for a test of whether or not observed effect sizes vary systematically according to assumed study level artifacts. Second, if there were a good (i.e., theoretically supported and reasonable) reason to control for a covariate within either the independent or dependent variable at the within-study 
level, then a semi-partial (i.e., "part") correlation may be derived on the basis of the zero-order relationships (See Cohen, Cohen, West \& Aiken, 2003, formula 3.3.8). Semi-partial correlations partition variance associated with a covariate from either the independent or dependent variable in a three-variable system. This approach has been used successfully in previous meta analyses to, for example, control for linear effects of age when meta-analytically summarizing estimates of curvilinear age-cognitive functioning relationships (see Verhaeghen \& Salthouse, 1997). Finally, and perhaps the best approach to addressing this issue, is to use either regression/path analysis or structural equation modeling (e.g., Cheung, 2008), which would allow for testing multiple plausible theoretical models with and without covariates controlled for in either the independent or dependent variable, or both.

Table 3. Meta "Mix" Results for Case \#1 \& Case \#2

\begin{tabular}{|c|c|c|c|}
\hline \multicolumn{4}{|c|}{ Case \#1 } \\
\hline $\begin{array}{c}\text { Mix Condition } \\
\text { (Correlations/Betas) }\end{array}$ & $\rho$ & 95\% CI Lower & 95\% CI Upper \\
\hline $10 / 90$ & 0.026 & 0.008 & 0.162 \\
\hline $20 / 80$ & 0.051 & 0.029 & 0.238 \\
\hline $30 / 70$ & 0.076 & 0.051 & 0.287 \\
\hline $40 / 60$ & 0.101 & 0.075 & 0.318 \\
\hline $50 / 50$ & 0.125 & 0.099 & 0.350 \\
\hline $60 / 40$ & 0.149 & 0.123 & 0.372 \\
\hline $70 / 30$ & 0.173 & 0.148 & 0.385 \\
\hline $80 / 20$ & 0.198 & 0.175 & 0.381 \\
\hline $90 / 10$ & 0.224 & 0.206 & 0.356 \\
\hline \multicolumn{4}{|c|}{ Case \#2 } \\
\hline $\begin{array}{c}\text { Mix Condition } \\
\text { (Correlations/Betas) }\end{array}$ & $\rho$ & 95\% CI Lower & 95\% CI Upper \\
\hline $10 / 90$ & 0.325 & 0.312 & 0.400 \\
\hline $20 / 80$ & 0.316 & 0.302 & 0.403 \\
\hline $30 / 70$ & 0.308 & 0.293 & 0.405 \\
\hline $40 / 60$ & 0.299 & 0.283 & 0.407 \\
\hline $50 / 50$ & 0.292 & 0.276 & 0.406 \\
\hline $60 / 40$ & 0.285 & 0.268 & 0.399 \\
\hline $70 / 30$ & 0.277 & 0.261 & 0.378 \\
\hline $80 / 20$ & 0.269 & 0.254 & 0.360 \\
\hline $90 / 10$ & 0.259 & 0.246 & 0.324 \\
\hline
\end{tabular}

Note. $\rho$ denotes meta-analytic effect size estimate. For each mix condition, $\mathrm{K}=100, \mathrm{~N}=25,000$

\section{Concluding Remarks.}

In summary, betas do not and should not rule meta-analyses. We have been cautioned against this practice in the past, and the conclusions of our simulation studies bolster this idea. The inclusion of even small percentages of partial relationships can have an undue effect on meta- 
analytic conclusions, and should thus not be considered by meta-analysts in any circumstances. In some respects, the simulations presented here are quite a narrow view of what may be a "worst case" scenario in a three-variable situation.

Figure 3. Meta "Mix" Results - Case \#1

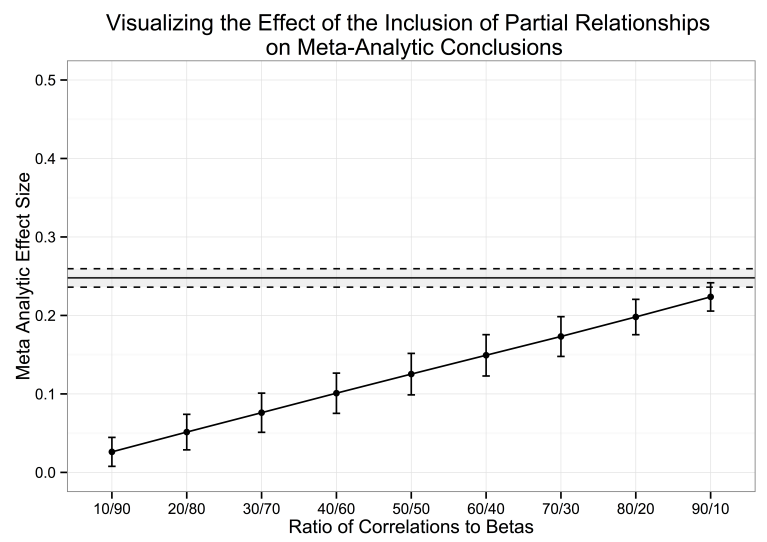

Figure 4. Meta "Mix” Results - Case \#2

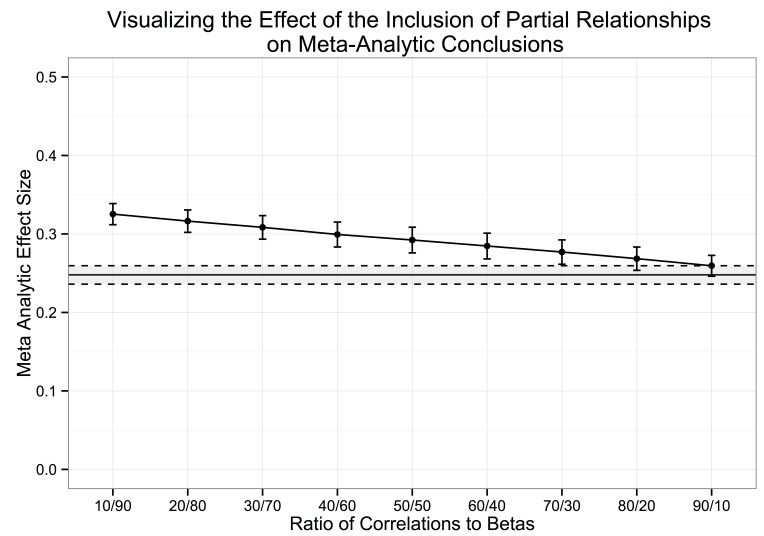

Note. Solid horizontal line indicates zero-order meta-analytic conclusion. Grey region bound by dashed lines indicates $95 \%$ confidence interval around this meta-analytic estimate. Error bars indicate $95 \%$ confidence interval for each meta-analytic estimate for the corresponding "mix" condition.

However, keep in mind that in real meta-analytic contexts, the covariate-independent variable and covariate-dependent variable relationships are rarely considered, and there are likely to be larger and more complex systems of dependencies that are at play in studies with multiple covariates. Of particular note, the equation presented above suggests that any non-zero covariateindependent variable relationship will yield partial relationships that diverge from zero-order relationships to some degree. Moreover, real-world studies rarely benefit from the random sampling procedure applied here. Further compounding the problem, bias is introduced by measurement error (i.e., unreliability) and issues of sample selection (e.g., range restriction) for each individual study. Considering all of the possible factors that serve as barriers to valid metaanalytic conclusions, the exclusion of partial relationships seems an easy point of remediation.

\section{References}

Becker, T. E. (2005). Potential problems in the statistical control of variables in organizational research: A qualitative analysis with recommendations. Organizational Research Methods, 8, 274-289.

Breaugh, J. A. (2008). Important considerations in using statistical procedures to control for nuisance variables in nonexperimental studies. Human Resource Management Review, 18, 282-293.

Cheung, M. W. L. (2008). A model for integrating fixed-, random-, and mixed-effects meta-analyses into structural equation modeling. Psychological methods, 13(3), 182202. 
Cohen, J., Cohen, P., West, S. G., \& Aiken, L. S. (2003). Applied multiple regression/correlation analysis for the behavioral sciences. Mahwah, NJ: Lawrence Earlbaum \& Associates.

Cooper, H. M.(2010). Research synthesis and meta-analysis. Thousand Oaks, CA: Sage Publications.

Ferguson, C. J. (2015a). Do Angry Birds make for angry children? A metaanalysis of video game influences on children's and adolescents' aggression, mental health, prosocial behavior, and academic performance. Perspectives on Psychological Science, 10(5), 646666.

Ferguson, C. J. (2015b). Pay no attention to that data behind the curtain: On angry birds, happy children, scholarly squabbles, publication bias, and why betas rule metas. Perspectives on Psychological Science, 10(5), 683-691.

Lipsey, M.W., \& Wilson, D. B. (2001). Practical meta-analysis. Thousand Oaks, CA: Sage Publications.

Meehl, P. E. (1971). High school yearbooks: A reply to Schwarz. Journal of Abnormal Psychology, 77, 143-148.

Paternoster, R. (1987). The deterrent effect of the perceived certainty of punishment: A review of the evidence and issues. Justice Quarterly, 4, 173-217.

Pratt, T., Cullen, F., Sellers, C., Winfree, T., Madensen, T., Daigle, L., . . Gau, J. M. (2010). The empirical status of social learning theory: A metaanalysis. Justice Quarterly, 27, 765802.

Savage, J., \& Yancey, C. (2008). The effects of media violence exposure on criminal aggression: A metaanalysis. Criminal Justice and Behavior, 35, 1123-1136.

Spector, P. E., \& Brannick, M. T. (2011). Methodological urban legends: The misuse of statistical control variables. Organizational Research Methods, 14, 287-305.

Hunter, J. E., \& Schmidt, F. L. (2015). Methods of meta-analysis: Correcting error and bias in research findings. Thousand Oaks, CA: Sage Publications.

Verhaeghen, P., \& Salthouse, T. A. (1997). Meta-analyses of age-cognition relations in adulthood: Estimates of linear and nonlinear age effects and structural models. Psychological bulletin, 122(3), 231-249. 